\title{
Characteristics of a stable arc based on FAST and MIRACLE observations
}

\author{
P. Janhunen ${ }^{1}$, A. Olsson ${ }^{2}$, O. Amm ${ }^{1}$, K. Kauristie ${ }^{1}$ \\ ${ }^{1}$ Finnish Meteorological Institute, Geophysical Research, Helsinki, Finland \\ ${ }^{2}$ Swedish Institute of Space Physics, Uppsala Division, Uppsala, Sweden
}

Received: 16 June 1999 / Revised: 11 October 1999 / Accepted: 14 October 1999

\begin{abstract}
A stable evening sector arc is studied using observations from the FAST satellite at $1250 \mathrm{~km}$ altitude and the MIRACLE ground-based network, which contains all-sky cameras, coherent radars (STARE), and magnetometers. Both FAST and STARE observe a northward electric field region of about $200 \mathrm{~km}$ width and a field magnitude of about $50 \mathrm{mV} / \mathrm{m}$ southward of the arc, which is a typical signature for an evening-sector arc. The field-aligned current determined from FAST electron and magnetometer data are in rather good agreement within the arcs. Outside the arcs, the electron data misses the current carriers of the downward FAC probably because it is mainly carried by electrons of smaller energy than the instrument threshold. Studying the westward propagation speed of small undulations associated with the arc using the all-sky cameras gives a velocity of about $2 \mathrm{~km} / \mathrm{s}$. This speed is higher than the background ionospheric plasma speed (about $1 \mathrm{~km} / \mathrm{s}$ ), but it agrees rather well with the idea originally proposed by Davis that the undulations reflect an $\mathbf{E} \times \mathbf{B}$ motion in the acceleration region. The ground magnetograms indicate that the main current flows slightly south of the arc. Computing the ionospheric conductivity from FAST electron data and using the ground magnetograms to estimate the current yields an ionospheric electric field pattern, in rather good agreement with FAST results.
\end{abstract}

Key words: Ionosphere (auroral ionosphere; ionosphere-magnetosphere interactions) -

Magnetospheric physics (auroral phenomena)

\section{Introduction}

Static or nearly static auroral arcs are a common feature in the auroral ionosphere, but there are still many open questions concerning the physical mechanisms responsible for their creation and maintenance. To make progress in the understanding of more dynamical cases, it is necessary to improve our knowledge of single isolated stable arcs, because many of the dynamical auroral phenomena such as curls, folds and auroral breakups may be at least partly described by perturbations of stable auroral arcs, or as waves propagating along the arcs.

The typical behavior of the horizontal electric field at and near to arcs in both evening and morning sectors is rather well known (Aikio, 1995; Marklund, 1984), but the distribution of the electric field at higher altitudes, especially above $10000 \mathrm{~km}$, is much less certain. There are indications that at least in some cases, the potential structure above inverted- $\mathrm{V}$ regions (Lin and Hoffman, 1979) could be O-shaped rather than U-shaped (Janhunen et al., 1999).

In this work we will study a FAST passage over a stable arc which is simultaneously detected by four ground-based all-sky cameras and the STARE bistatic coherent radar. The instrumentation allows us to compare the optical and satellite-inferred widths of the arcs and to compare the horizontal electric fields as measured by the satellite and by the radars. Likewise, we estimate the westward velocity of horizontal arc undulations from the all-sky images and compare this with the electric field measurements and discuss it also in terms of a model in which these undulations propagate with an $\mathbf{E} \times \mathbf{B}$ velocity persisting higher up in the acceleration region (Haerendel et al., 1996).

\section{Instrumentation}

The FAST satellite (Carlson et al., 1998a) was launched in August 21, 1996 into an $83^{\circ}$ elliptic orbit with an apogee of $4175 \mathrm{~km}$ and a perigee of $350 \mathrm{~km}$. DC electric and magnetic field data as well as the electron data from FAST are used in this study.

We will use data from the MIRACLE (Magnetometer, Ionospheric Radar and All-sky Camera Large Experiment) ground-based instrument network (Syrjäsuo et al., 1998) (Fig. 1). The all-sky camera (ASC) data from the three stations at Kilpisjärvi (KIL), Kevo 
(KEV) and Muonio (MUO) have a time resolution of $20 \mathrm{~s}$. A $557 \mathrm{~nm}$ filter was used, thus the images show only green aurora. The $630 \mathrm{~nm}$ filter images recorded at $60 \mathrm{~s}$ interval are not used in this study. We will also use the electric field observations of the recently renewed STARE (Scandinavian Twin Auroral Radar Experiment) radar (Greenwald et al., 1978, Nielsen et al., 1998) and refer to the IMAGE magnetometer network (Lühr et al., 1998) to establish that the level of geomagnetic disturbances is rather low in this event.

\section{Observations}

On November 3 1998, 17:35-17:37 UT, FAST moved from north to south above northern Scandinavia detecting two rather narrow and moderately intense (about $10 \mathrm{~mW} \mathrm{~m}^{-2}$ ) inverted-V type precipitation regions. The FAST electron spectrograms are shown in Fig. 2. We have performed Maxwellian fits to these spectra (not shown). The peak electron energy within the arcs is around $5 \mathrm{keV}$ and the characteristic energy (temperature) about $1.5 \mathrm{keV}$. As can be seen in Fig. 2, FAST passes the first one at about 17:35:37-17:35:45 UT and the second one at 17:36:11-17:36:18 UT. We call these arcs arc 1 and arc 2, respectively. In between arcs 1 and 2 there are also weaker subvisual arcs. We

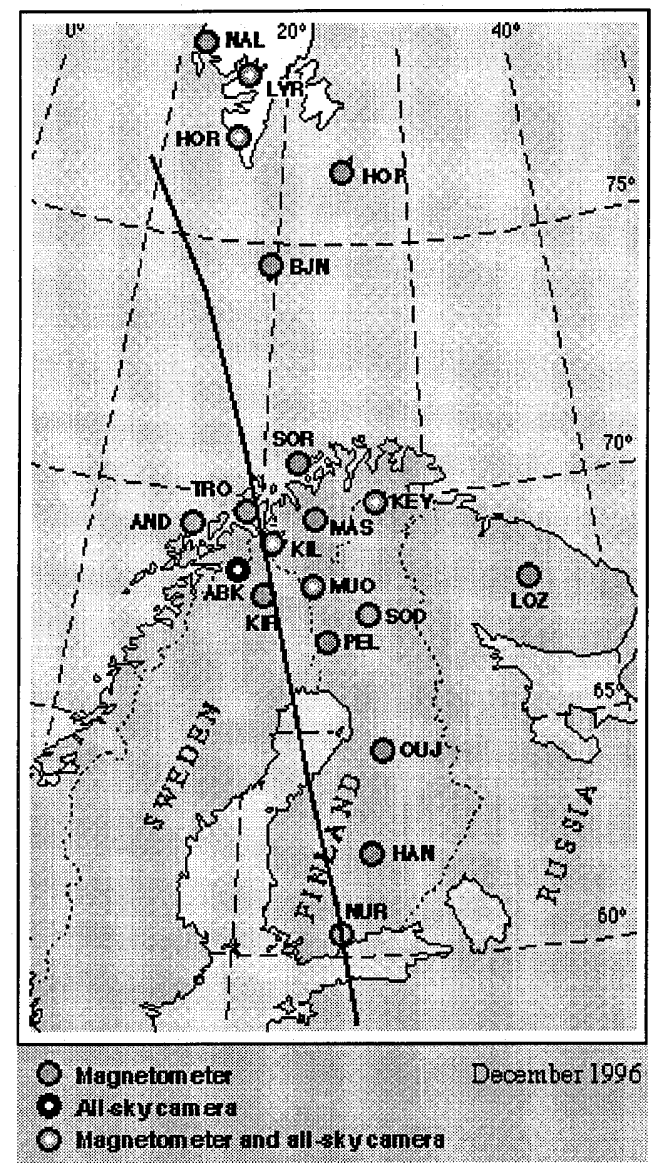

Fig. 1. Locations of MIRACLE all-sky camera and magnetometer stations together with the ionospheric projection of the FAST orbit want to emphasize that although arc 1 looks very similar to arc 2 in the FAST data, it does not seem to be an elongated arc according to the ASC recordings at KIL. It is rather a single "blob" of precipitation, or if it is an arc, it is generally less intense than arc 2 but has a local enhancement just at the point where FAST passes through. Since the "blob" is not a temporally stable feature, in our later analysis we will mostly concentrate on arc 2 which is a typical stable arc.

In Fig. 3 we show the field-aligned current (FAC) as determined by two different methods from FAST data, using the magnetic field variation and electron data. The ion contribution to the FAC is found from the FAST ion data (not shown) to be negligible in this case. The inferred upward (positive) FAC density of a few $\mu \mathrm{A} \mathrm{m} \mathrm{m}^{-2}$ agrees well between the two methods. The satellite magnetometer shows that outside the arcs, there is a downward (negative) FAC, not visible in the electron data (the FAC estimated from the electron detector is positive throughout the event), probably because FAST is at $1250 \mathrm{~km}$ altitude and thus the downward current is carried by upward moving cold electrons which are below the instrument threshold $(25 \mathrm{eV})$. In regions where the magnetometer shows a downward FAC the electron precipitation is small, except around $69.5^{\circ}$ GLAT where there is a secondary peak in the upward FAC carried by precipitating electrons. The secondary peak is correlated with an upward excursion of the magnetometer-produced FAC. This is a signature of counterstreaming electrons.

The passing time as determined from FAST (Fig. 2) for arc 2 is about $7 \mathrm{~s}$. The velocity of the spacecraft is $7.6 \mathrm{~km} / \mathrm{s}$, giving the arc width at FAST altitude of about $53 \mathrm{~km}$, which at ionospheric altitude corresponds to about $40 \mathrm{~km}$ (using the dipole approximation).

The MIRACLE magnetometer and all-sky camera stations were shown together with the footprint of the FAST orbit in Fig. 1. We use data from the KIL, MUO and KEV all-sky cameras. The Abisko (ABK) all-sky camera also detected the arcs, but the viewing conditions were not so good. The KIL station is exactly below the FAST trajectory, and MUO is close to it. Of these, the KIL station has the best viewing angle of the arc. The KEV station has excellent viewing conditions and the arc is close to zenith, but it views the arc about $300 \mathrm{~km}$ east of the FAST footpoint.

Both the MUO and KIL recordings show that arc 2 is centered at $69.9^{\circ} \pm 0.1^{\circ}$ geographic latitude. The IMAGE magnetometer data shows that the level of magnetic activity is low $(<100 \mathrm{nT})$. From a close inspection of the all-sky images at different times it is found that arc 2 hardly moves in the north-south direction during the event.

In Fig. 4 we show the KIL all-sky camera image at 17:36:20 histogram-equalized, false-colored and mapped to $110 \mathrm{~km}$ altitude. The histogram equalization makes it easier to detect the location of the arc while making the information about exact intensities less representative.

From the all-sky cameras we can conclude that the arc width is less than $30 \mathrm{~km}$, which is slightly less than 


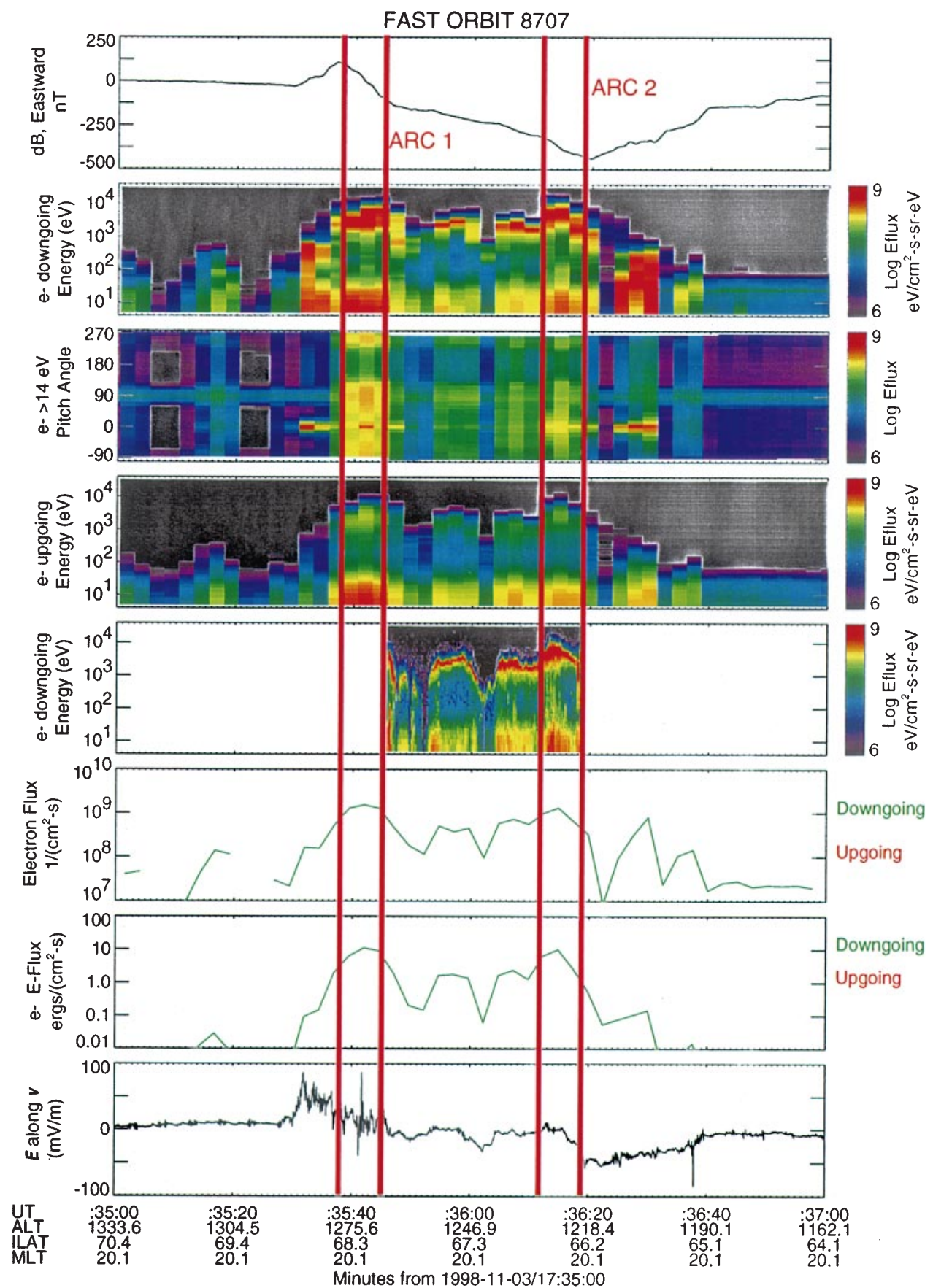

Fig. 2. FAST data for 19981103 , 17:35-17:37 UT. Panels from top to bottom are numbered from 1 to 8. 1, Eastward magnetic field deflection from the satellite magnetometer; 2, downgoing electron energy flux; 3 , pitch angle distribution of electrons, 4 , upgoing electron energy flux, 5 , burst mode data similar to panel $2 ; 6$, total electron flux; 7 , total electron energy flux; 8 , electric field component along spacecraft orbit. Arcs identified from FAST electrons are marked with red lines. In panels 6 and 7, no red curves are visible because the electron flux is downgoing throughout the event the FAST estimate of $40 \mathrm{~km}$ given above. However, the half-value width of the energy flux peak of arc 2 (Fig. 2, panel 7) is about $5 \mathrm{~s}$, which corresponds to a spatial distance of $30 \mathrm{~km}$ at the ionosphere. Thus the optical and FAST widths are not in disagreement.

We now consider the equatorward side of arc 2. In this region the electric field is, according to STARE, about $50 \mathrm{mV} / \mathrm{m}$ (corresponding to about $1 \mathrm{~km} / \mathrm{s}$ electron flow velocity) and pointing northward. Generally, STARE receives an echo only when the electric field is above the Farley-Buneman threshold. The threshold value depends on the E-region ion temperature but is usually about $17 \mathrm{mV} / \mathrm{m}$. Near the boundaries of the region where STARE receives echoes we indeed see that the electric fields are of the order of $17 \mathrm{mV} / \mathrm{m}$. STARE flow velocity vectors are drawn in Fig. 5 only when both radars' backscatter intensity was at least $2 \mathrm{~dB}$ above the background noise.

FAST electric field data (Fig. 7) also show a northward electric field with a maximum of $50 \mathrm{mV} / \mathrm{m}$ and an average of $40 \mathrm{mV} / \mathrm{m}$ on the southern side of arc 2, in accordance with STARE. Mapped to ionosphere, $40 \mathrm{mV} / \mathrm{m}$ corresponds to $50 \mathrm{mV} / \mathrm{m}$ so the agreement with STARE is good. Between the arcs the FAST electric field is weak, below the STARE threshold. On the northern side of arc 1 FAST sees a narrow region of high southward electric field, which is outside the STARE field of view, however. 
FAST FAC

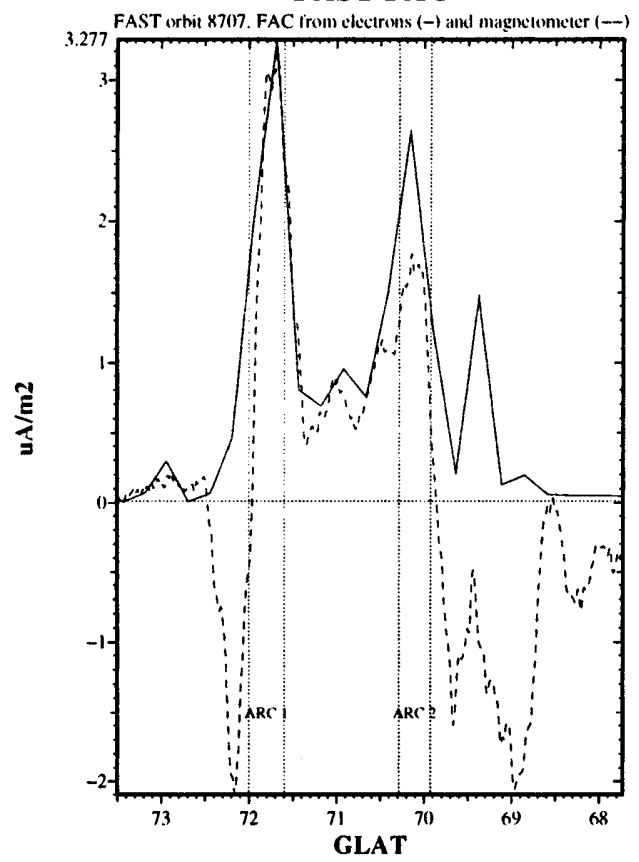

Fig. 3. FACs determined from FAST magnetic field and particle data plotted versus geographic latitude. Positive FAC is upward. The solid line represents the FAC computed from the electron data while the dashed line is the FAC estimated from the satellite magnetometer data

The FAST traversal is close to the westward boundary of the STARE field of view. About $200-300 \mathrm{~km}$ east of the FAST trajectory, STARE detects a northward electric field also on the northern side of arc 2 ,

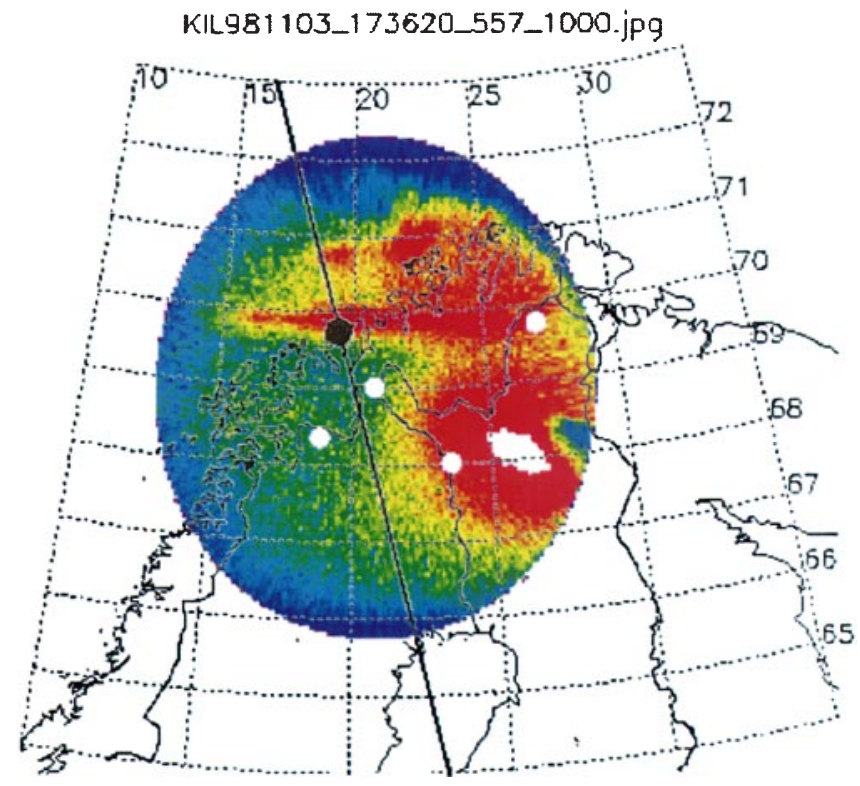

Fig. 4. Histogram-equalized false color image, mapped to $110 \mathrm{~km}$ altitude of the Kilpisjärvi $(K I L)$ all-sky camera (the white dot at the center of the circle) together with FAST trajectory and position (black dot) at 17:36:20 UT on November 3, 1998. We see that arc 2 is centered at about $70^{\circ}$ geographic latitude and its width, as deduced from the all-sky camera is $30 \mathrm{~km}$ or smaller. The bright area dominating the southeastern part of the all-sky image is the Moon while the field seen by FAST is much weaker. We do not know an exact reason for this. One possibility is that the electric field north of the arc changes in the east-west direction.

Between arcs 1 and 2 the satellite-measured electric field is anticorrelated with the electron precipitation quite well. (The region is filled with weak subvisual arcs.) Comparing panel 6 of Fig. 2 with the lower panel of Fig. 7 we see that whenever there is a peak in the precipitating energy flux, there is a plateau in the potential. At arc 1, which is a precipitation blob rather than a real arc, no plateau is seen.

\section{Discussion and conclusions}

\subsection{Comparisons with previous work}

FAST electron data miss the downward field-aligned current in this case. In other studies where this did not happen (Carlson et al., 1998b), FAST was always close to $4000 \mathrm{~km}$ altitude, i.e., at regions where the electrons had gained enough energy from a downward electric field to be detected.

To check that our explanation for the downward current carriers is a feasible one we can estimate how large electron density is required to carry the required downward current density by electrons whose upward speed does not exceed the instrument threshold, i.e., $j=e n v$ with $(1 / 2) m_{e} v^{2}<25 \mathrm{eV}$. For $j=2 \mu \mathrm{A} \mathrm{m}^{-2}$ (the largest current, see Fig. 3) we obtain $n>4.2 \mathrm{~cm}^{-3}$. Typical densities at the $1200 \mathrm{~km}$ altitude are much higher, so cold electrons can very well act as current carriers and still remain undetected.

The FAC structure for arc 2 (upward FAC on the northern side of a downward one) is in accordance with a schematic model of evening sector arcs (Fig. 6, taken from Aikio, 1995).

Optical widths of arcs as compared to FAST have recently been analyzed using TV-cameras mounted on an aircraft (Stenbaek-Nielsen et al., 1998). Our all-sky camera data do not allow a detailed comparison.

In the classification of Marklund (1984), arc 2 is most likely an evening anticorrelation polarization arc (type Ia).

\subsection{Davis-Haerendel model}

When viewing the all-sky images as a movie with suitable color corrections, it is possible to see that there are westward-propagating small undulations or weak bulges. By determining the position of these structures in the adjacent KIL frames ( $20 \mathrm{~s}$ time resolution) from the images mapped to the geographic grid we can estimate that the westward velocity is in the range $1.3-3 \mathrm{~km} / \mathrm{s}$, the most probable value being $2 \mathrm{~km} / \mathrm{s}$ when FAST passes over. The uncertainty is mainly due to an apparent temporal variability of the propagation speed. We believe that these are the same phenomena that have been observed earlier using TV cameras (Davis, 1978; 
MIRACLE

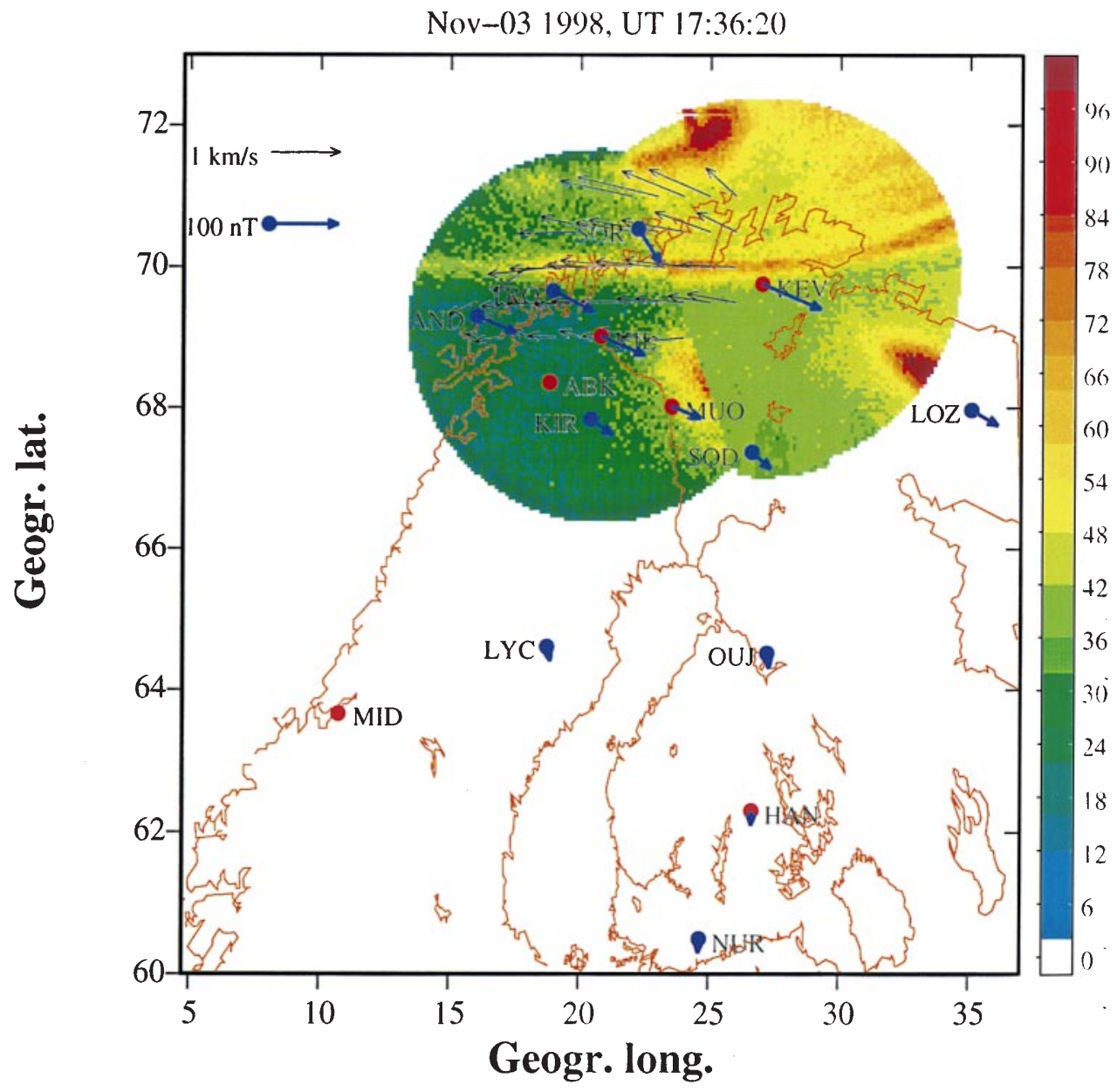

FNI/R:1:O

Fig. 5. MIRACLE plot showing $K I L$ and $K E V$ all-sky camera views mapped to $110 \mathrm{~km}$ altitude, STARE electron velocity vectors (black) and IMAGE ground magnetometer equivalent current vectors (dark blue) 17:36:20 UT. The color scaling corresponds to ASC pixel values (originally in the range $0-255$ ). The red spots in the southeast are images of the Moon. Arc 2 extends through both camera views close to $70^{\circ}$ geographic latitude. STARE shows a westward drift of about $1 \mathrm{~km} / \mathrm{s}$ (corresponding to a northward electric field of about $50 \mathrm{mV} / \mathrm{m}$ )
Haerendel et al., 1996). They suggested that the velocity of the undulations would correspond to the $\mathbf{E} \times \mathbf{B}$ velocity, where $E$ is associated with the "sides" of a U-shaped potential in the acceleration region (of course, mapped down to the ionosphere to honor the flux tube convergence). This model uses the assumption that the parallel potential drop $V_{0}$ estimated from the lowaltitude electron peak energy $\left(V_{0} \approx 5 \mathrm{kV}\right.$ for arc 2$)$ is the same as the perpendicular potential drop associated with the "side" of the U-shaped potential (Fig. 8).

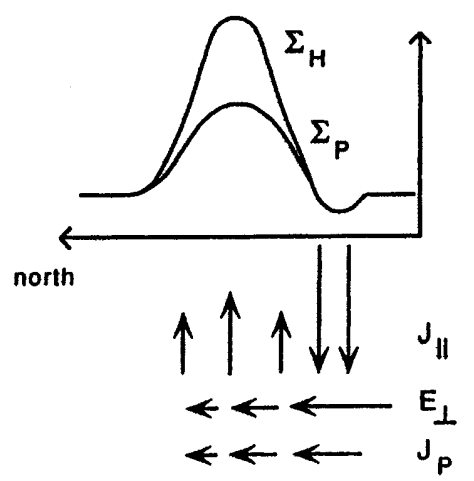

Fig. 6. A schematic model of an evening-sector arc (from Aikio, 1995)
To apply the Davis-Haerendel idea in our event, we note that the $\mathbf{E} \times \mathbf{B}$ velocity at any point, mapped down to the ionosphere, is $v_{i}=\sqrt{B / B_{i}}(E / B)=E / \sqrt{B_{i} B}$. For each latitude point, we find the maximum of $v_{i}$ along the field line and call it the Davis-Haerendel velocity $v_{D H}$. We can do this in both O- and U-shaped potential models (Janhunen et al., 1999), but the result is practically the same in both cases. We show the result in Fig. 9. In regions where there is not enough inverted-V type precipitation, $v_{D H}$ is not a sensible quantity and those points have not been plotted in Fig. 9.

We see that inside the equatorward half of $\operatorname{arc} 2, v_{D H}$ attains a maximum of about $4 \mathrm{~km} / \mathrm{s}$, but is about $2 \mathrm{~km} / \mathrm{s}$ in a region just adjacent to arc 2 . It is in the adjacent region that the speed of the undulations was measured; thus we conclude that the Davis-Haerendel model produces an estimate which may be somewhat higher than the observed value, but taking into account the observational uncertanties there is no disagreement between the two.

\subsection{FAC determined from ground-based measurements}

Instantaneous, two-dimensional distributions of ionospheric electrodynamic parameters (i.e., height-integrat- 

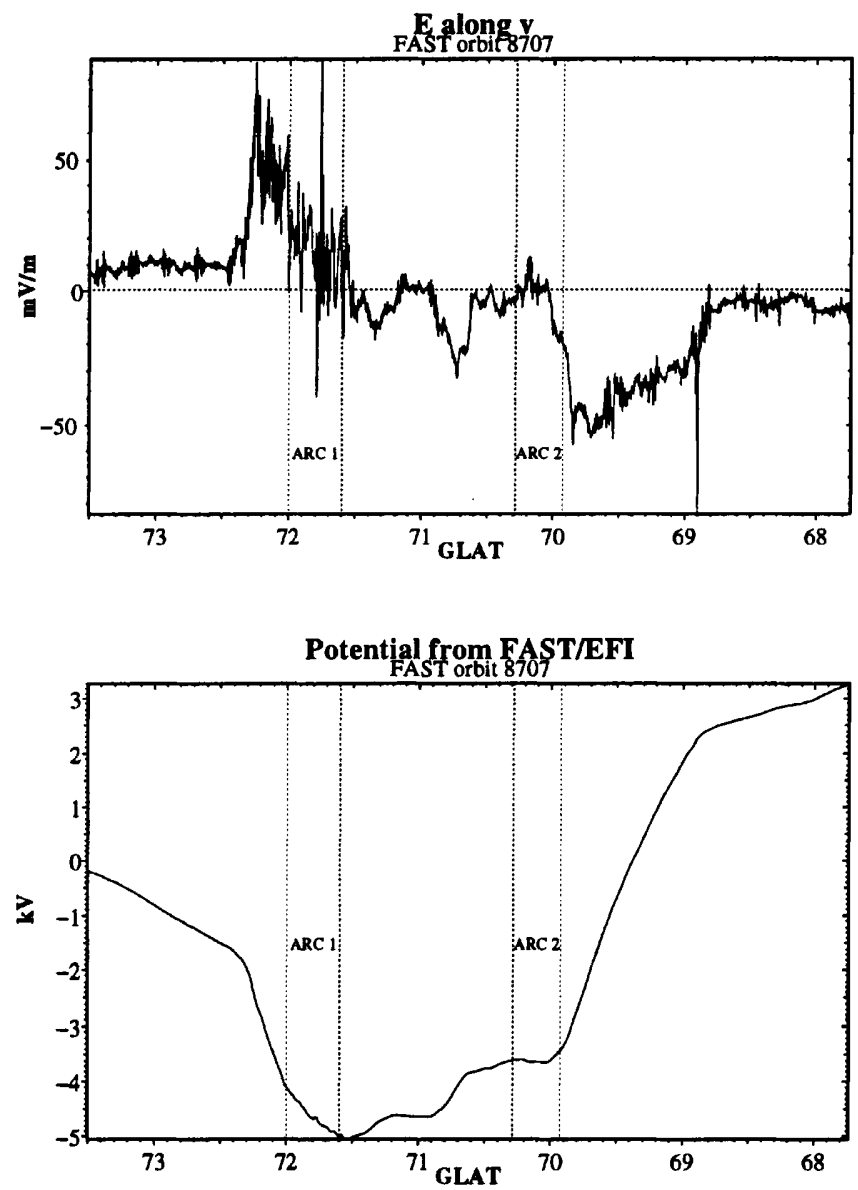

Fig. 7. FAST electric field along spacecraft orbit (upper panel) and integrated potential along the orbit (lower panel)

ed Hall and Pedersen conductances, and horizontal and field-aligned currents) can be obtained from groundbased measurements of the magnetic field disturbance and coherent scatter radar observations of the ionospheric electric field using the method of characteristics (Amm, 1998). In addition to the measurements, an estimate of the Hall- to Pedersen conductance ratio is needed. However, the effect of this estimate on the final results has been shown to be small in most cases (Amm, 1995). Moreover, the value of the conductance ratio can be assessed from the ground magnetic disturbance level (e.g., Lester et al., 1996). For the present event, the STARE radar did not provide sufficient backscatter to apply a full $2 \mathrm{D}$ analysis. Thus, and since the arc under study is essentially a 1D structure, we apply a onedimensional version of the method of characteristics which yields the same output quantities on a northsouth profile (see Inhester et al., 1992).

The FAC distribution resulting from this analysis, carried out in the latitude range between 69 and $71^{\circ}$, is shown in Fig. 10, together with the FAC obtained from the FAST magnetometer. Both results show considerable upward FAC in the vicinity of arc 2. However, the magnitude of the FAC is considerably larger when inferred from the ground-based data than from the FAST data, and its peak is shifted slightly towards

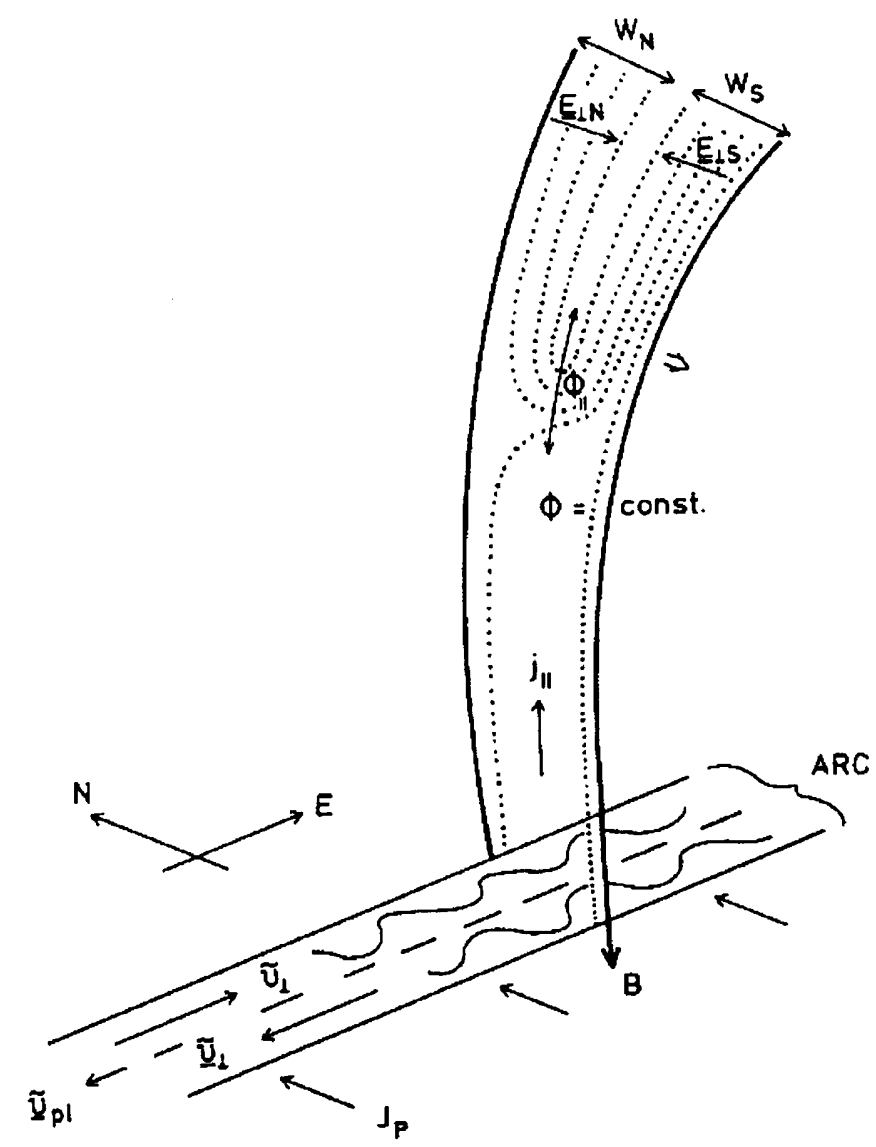

Fig. 8. A schematic figure of the Davis-Haerendel suggestion to explain the high speed of east-west undulations which propagate along the edges of an arc (reproduced from Haerendel et al., 1996, Fig. 8)

the south. One reason for these differences can be the smaller spatial resolution of the ground-based data which is limited to about $50 \mathrm{~km}$ for ground magnetometers. Another possibility is that the arc may not be completely uniform in the east-west direction, since the ground-based profile is located about $4^{\circ}$ of longitude

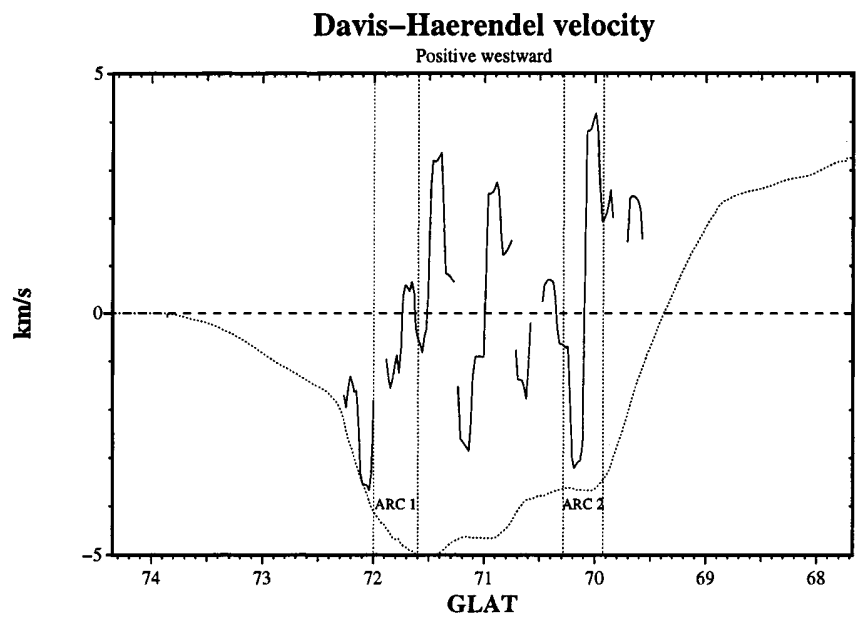

Fig. 9. The perpendicular velocity according to Davis-Haerendel suggestion, applied to our event. The FAST potential from Fig. 7 (in $\mathrm{kV}$ ) is shown as a visual aid (dotted line) 


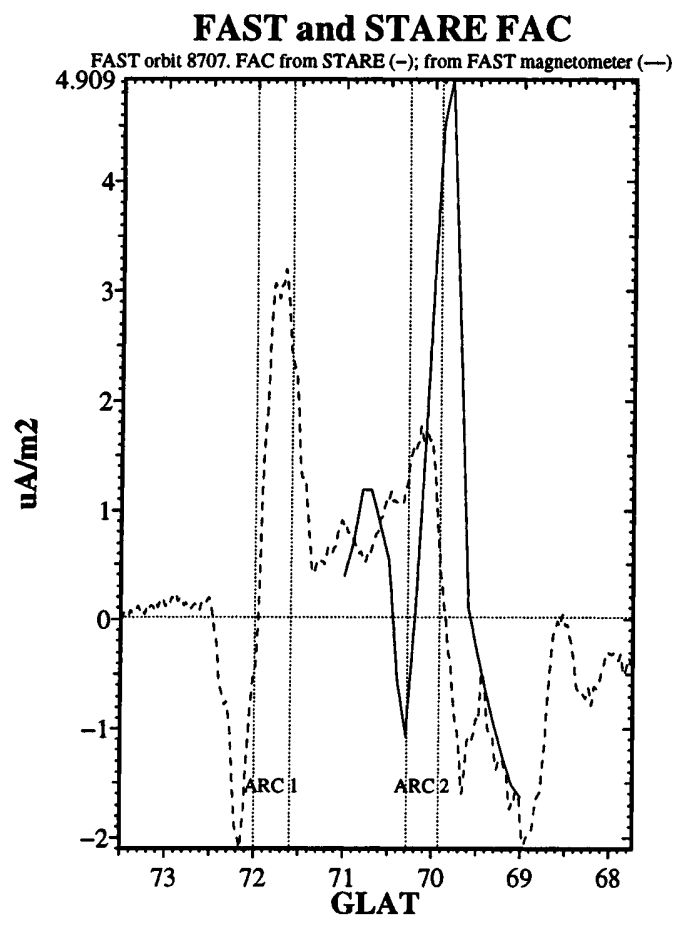

Fig. 10. The FAC determined from STARE and ground magnetometers by the method of characteristics (solid line) is compared here with the FAC determined from FAST magnetometer (dashed line). Positive FAC is upward

eastward of the FAST satellite path. Another discrepancy between the FAST and the ground-based data is that the magnetometers show the largest eastward electrojet (equivalent) currents not at arc 2, but 50 $100 \mathrm{~km}$ south of it; this can be seen e.g., from the location of zero of interpolated and upward-continued vertical variation magnetic field component (not shown). Accordingly, to carry this current, a moderate conductance $(5-10 \mathrm{~S})$ required in this region results from the analysis with the method of characteristics (data not shown), although the electron precipitation detected by FAST is weak there. It should be noted that such an electrojet flow south of an optical arc is a typical situation in ground-based observations in the evening sector (Marklund et al., 1982). One possible explanation for the origin of the conductance south of the arc is that it is caused by proton precipitation. According to FAST data (not shown), in this region the ion energy flux is still increasing when the upper limit of the energy range $(25 \mathrm{keV})$ is reached; thus the magnetospheric protons are hot and could provide significant ionization that results in the observed conductance values. The ionization production profiles by proton and electron precipitation are known (Kirkwood and Osepian, 1995), but since we do not have measurements of high energy ions, we cannot study this quantitatively here. More loworbiting satellite/ground-based conjunction studies are needed to resolve the difference between the conductance distribution as obtained by ground-based measurements, and the one expected from the satellite's precipitating electron measurements.
An alternative approach is to solve the electric field from the ionospheric electrodynamic equations, if the ground magnetograms and the ionospheric conductivities are known. We can determine the conductivities from FAST electron data using the formulas given by (Robinson et al., 1987). The computed conductivities are shown in the top panel of Fig. 11. The resulting electric field is shown in the bottom panel, together with the northward electric field measured by FAST. Taking into account the observational inaccuracies the agreement is rather good. The discrepancy in the electric field in the 69.0-69.5 geographic latitude could be reduced by assuming that the conductivities are slightly enhanced here e.g., due to proton precipitation. The STARE electric field cannot be directly compared with this since STARE has only a very few data points which are exactly under the FAST trajectory. If one averages the STARE northward electric field in the east-west direction, one obtains a result which is markedly different from the FAST or the Robinson-model curves: the STARE field is not small north of and especially inside arc 2 . We do not have a good explanation for this, other than that STARE
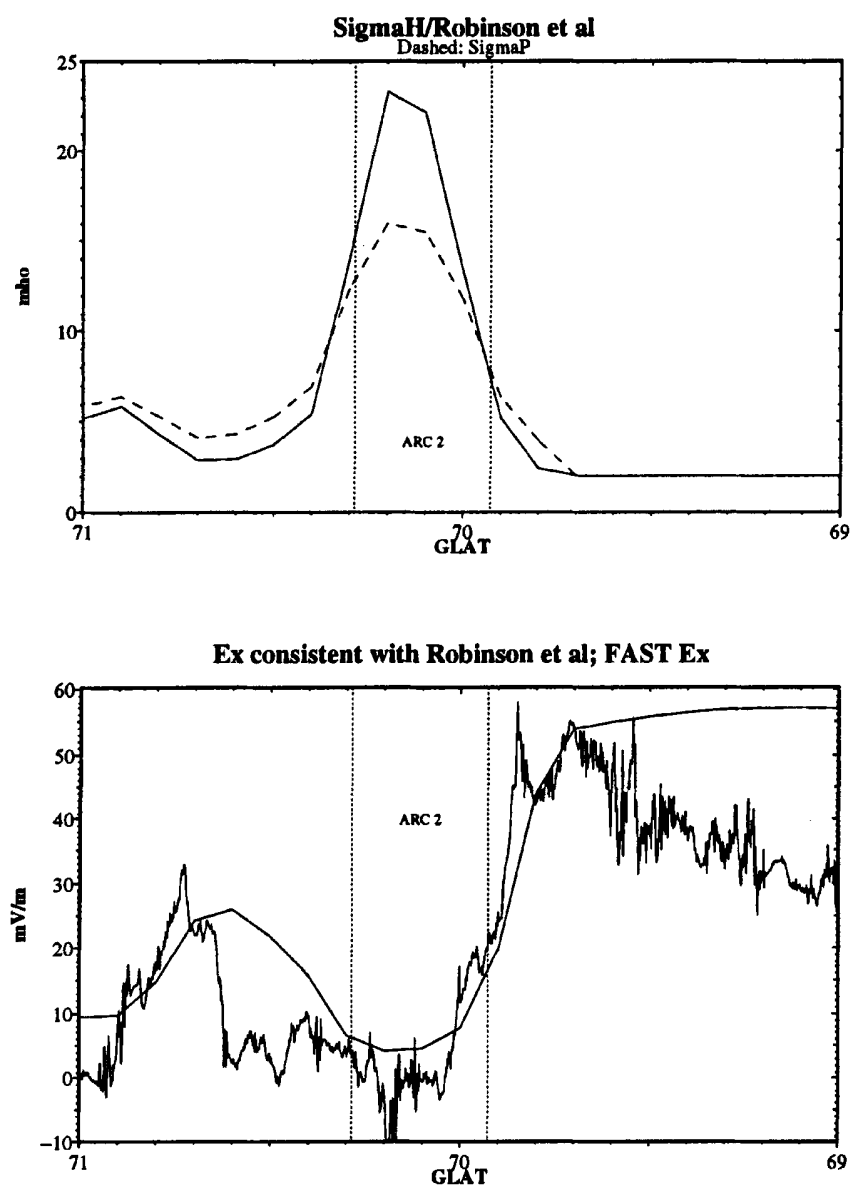

Fig. 11. Top, height-integrated ionospheric Hall (solid line) and Pedersen (dashed) conductivity as determined from FAST electron data using formulas of Robinson et al. (1987). Conductivities smaller than 2 mho have been set to 2 mho. Bottom, northward electric field inferred from the ground magnetograms and the conductivities in the top panel (smoother curve), and FAST northward electric field (jagged curve) 
is not measuring exactly the same region and that the arc may be too narrow for STARE to resolve. To figure out a possible cause for the discrepancy, it would be worthwhile in the future to try to find more exact arc conjunctions between FAST and STARE.

\section{Conclusions}

1. A stable arc (called arc 2 in the text) was studied using FAST and MIRACLE. In Marklund's classification, the arc is most likely an evening anticorrelation polarization arc.

2. FAST gives an estimate of $30 \mathrm{~km}$ for the width of the arc (the half-value width of the precipitating energy flux). This is the same as the upper limit for the width given by the all-sky camera data.

3. STARE and FAST agree upon the northward electric field region southward of the arc. This region is a few hundred $\mathrm{km}$ wide and the typical electric field is $50 \mathrm{mV} / \mathrm{m}$ in this case. This is a typical feature of an evening-sector arc (Aikio et al., 1993).

4. The field-aligned current (FAC) determined from FAST electron data and magnetometer are in rather good agreement within the arcs. Outside the arcs, the electron data misses the downward FAC probably because it is carried by electrons that have smaller energy than the instrument threshold. This is not strange since FAST is only at $1250 \mathrm{~km}$ altitude for this event.

5 . The westward speed of small undulations of the arc (about $2 \mathrm{~km} / \mathrm{s}$ ) is larger than the background $\mathbf{E} \times \mathbf{B}$ velocity $(1 \mathrm{~km} / \mathrm{s})$. Assuming that the speed would be generated within the acceleration region using the Davis-Haerendel idea would give about $2-4 \mathrm{~km} / \mathrm{s}$ speed depending on the exact position, thus this idea seems to fit in our event, within the observational uncertainties.

6. Solving the ionospheric electrodynamics using ground-based data with the one-dimensional method of characteristics gives a FAC pattern which peaks in the vicinity of arc 2.

7. Ground magnetometer data indicate that the main eastward electrojet current flows $50-100 \mathrm{~km}$ south of arc 2. This requires a higher conductivity than that produced by electron precipitation. Possibly, the enhanced conductivity could be due to proton precipitation.

8 . By computing the ionospheric conductivities from FAST electron data and using ground magnetometers to estimate the current, the resulting ionospheric electric field pattern is in rather good agreement with FAST. The pattern differs from that inferred from STARE especially at the arc. This may be due to the fact that the region where STARE receives enough backscatter in this event does not exactly coincide with the FAST trajectory or that the arc is too narrow for STARE to resolve.

Acknowledgements. We thank J.P. McFadden and C.W. Carlson for providing the FAST data and for giving valuable interpretation hints. We thank all the participating institutes of the IMAGE magnetometer network. The work of A.O. was partly supported by the Knut and Alice Wallenberg Foundation and the Swedish Natural Science Research Council. The work of O.A. was supported by a grant from the German Science Foundation, and by a DAAD postdoctoral fellowship HSP III financed by the German Federal Ministry for Research and Technology.

Topical Editor G. Chanteur thanks G. Marklund and A. D. Aylward for their help in evaluating this paper.

\section{References}

Aikio, A., Studies of the electrodynamics of the auroral oval, Ph D Thesis, Department of Physical Sciences, University of Oulu, Finland, 1995.

Aikio, A., H. J. Opgenoorth, M. A. L. Persson, and K.U. Kaila, Ground-based measurements of an arc-associated electric field, J. Atmos. Terr. Phys., 55, 797-808, 1993.

Amm, O., Direct determination of the local ionospheric Hall conductance distribution from two-dimensional electric and magnetic field data: application of the method using models of typical ionospheric electrodynamic situations, J. Geophys. Res., 100, 21473-21488, 1995.

Amm, O., Method of characteristics in spherical geometry applied to a Harang discontinuity situation, Ann. Geophysicae, 16, 413424, 1998

Carlson, C. W., R. F. Pfaff, and J. G. Watzin, The Fast auroral snapshot (FAST) mission, Geophys. Res. Lett., 25, 2013-2016, 1998a.

Carlson, C. W., J. P. McFadden, R. E. Ergun, M. Temerin, W. Peria, F. S. Mozer, D. M. Klumpar, E. G. Shelley, W. K. Peterson, E. Moebius, R. Elphic, R. Strangeway, C. Cattell, and R. Pfaff, FAST observations in the downward auroral current region: energetic upgoing electron beams, parallel potential drops, and ion heating, Geophys. Res. Lett., 25, 2017-2020, 1998 b.

Davis, T. N., Observed characteristics of auroral forms, Space Sci. Rev., 22, 77-113, 1978.

Greenwald, R. A., W. Weiss, E. Nielsen, and N. R. Thomson, STARE: a new radar auroral backscatter experiment in northern Scandinavia, Radio Sci., 13, 1021-1039, 1978.

Haerendel, G., B. U. Olipitz, S. Buchert, O. H. Bauer, E. Rieger, and C. La Hoz, Optical and radar observations of auroral arcs with emphasis on small-scale structures, J. Atmos. Terr. Phys., 58, 71-83, 1996.

Inhester, B., J. Untiedt, M. Segatz, and M. Kürschner, Direct determination of the local ionospheric Hall conductance distribution from two-dimensional electric and magnetic field data, J. Geophys. Res., 97, 4073-4083, 1992.

Janhunen, P., A. Olsson, F. S. Mozer, and H. Laakso, How does the U-shaped potential close above the acceleration region? A study using Polar data, Ann. Geophysicae, in press, 1999.

Kirkwood, S., and A. Osepian, Quantitative studies of energetic particle precipitation using incoherent-scatter radar, J. Geomagn. Geoelectr., 47, 783-799, 1995.

Lester, M., J. A. Davies and T. S. Virdi, High-latitude Hall and Pedersen conductances during substorm activity in the SUNDIAL-ATLAS campaign, J. Geophys. Res., 101, 26 719-267 28, 1996.

Lin, C. S, and R. A. Hoffman, Characteristics of the inverted-V event, J. Geophys. Res., 84, 1514-1525, 1979.

Lühr, H., A. Aylward, S. C. Bucher, A. Pajunpää, K. Pajunpää, T. Holmboe, and S. M. Zalewski, Westward moving dynamic substorm features observed with the IMAGE magnetometer network and other ground-based instruments, Ann. Geophysicae, 16, 425-440, 1998.

Nielsen, E., M. Bruns, I. Pardowitz, H. Perplies, L. Bemmann, P. Janhunen, and A. Huuskonen, STARE: observations of a field-aligned line current, Geophys. Res. Lett., 26, 21-24, 1999.

Marklund, G., I. Sandahl, and H. Opgenoorth, A study of the dynamics of a discrete auroral arc, Planet. Space Sci., 30, 179197, 1982.

Marklund, G., Auroral arc classification scheme based on the observed arc-associated electric field pattern, Planet. Space Sci., 32, 193-211, 1984. 
Robinson, R. M., R. R. Vondrak, K. Miller, T. Dabbs, and D. Hardy, On calculating ionospheric conductances from the flux and energy of precipitating electrons, J. Geophys. Res., 92, 25652569, 1987.

Stenbaek-Nielsen, H. C., T. J. Hallinan, D. L. Osborne, J. Kimball, C. Chaston, J. McFadden, G. Delory, M. Temerin, and C. W. Carlson, Aircraft observations conjugate to FAST: auroral arc thickness, Geophys. Res. Lett., 25, 2073-2076, 1998.
Syrjäsuo, M. T., T. I. Pulkkinen, P. Janhunen, A. Viljanen, R. J. Pellinen, K. Kauristie, H. J. Opgenoorth, S. Wallman, P. Eglitis, P. Karlson, O. Amm, E. Nielsen, and C. Thomas, Observations of substorm electrodynamics by using the MIRACLE network, Substorms-4 (Proc. ICS-4 at Nagoya), Terra, Tokyo, 1998. 